\title{
Enumeration of A Family of Perfect Quaternary Arrays
}

\author{
Fanxin Zeng \\ Chongqing Key Laboratory of Emergency \\ Communication, Chongqing Communication Institute \\ Chongqing 400035, China \\ e-mail: fzengx@gmail.com,

\section{Zhenyu Zhang} \\ Chongqing Key Laboratory of Emergency \\ Communication, Chongqing Communication Institute \\ Chongqing 400035, China \\ e-mail: emzhenyuzhang@ hotmail.com,
}

\begin{abstract}
This paper investigates the enumeration of a family of perfect quaternary arrays (PQAs) from Zeng, et al's constructions. By deriving the conditions which result in distinct PQAs, the family size of Zeng, et al's constructions is determined, and distinct PQAs are educed from the obtained conditions. Finally, two examples are given. The proposed distinct PQAs provide lots of candidates for applications to communications, radar, and so on.
\end{abstract}

Keywords-perfect quaternary array, periodic autcorrelation, distinct array, family size, $n$-dimensional array

\section{INTRODUCTION}

A perfect array means a $n$-dimensional function whose autocorrelation is impulse-like. Perfect arrays are widely applied to high-dimensional communications, time-frequency-coding, spatial correlation or map matching, built-in tests of VLSI-circuits, coded aperture imaging, phased array antennas, arrays of sound sources, radar, and so on [1]-[3]. In the existing literature, there are a large number of perfect binary arrays (PBAs) [3]-[9], perfect ternary arrays (PTAs) [10] [11], and perfect multiphase arrays [12]. However, the constructions of perfect quaternary arrays (PQAs) only have a few. To the best of the author's knowledge, Arasu and Launey gave a family of PQAs by making use of polynomial theory [13], and Zeng, et al proposed a method converting a PBA into a PQA [14]. With regard to the advances on perfect arrays, please refer to [15].

This paper follows [14] so as to solve the problem of the enumeration of Construction 2 in it. By deriving the existence conditions of distinct PQAs in Construction 2 in [14], this paper determines the family size of Construction 2, and distinct PQAs are educed from the obtained conditions. More clearly, for given two $n$ dimensional PQAs with size $N_{1} \times \cdots \times N_{k-1} \times N_{k} \times$ $N_{k+1} \times \cdots \times N_{n}$, where the positive integer $N_{k}$ is odd, the family size of Construction 2 arrives at $N_{k}$, in other

\author{
Linjie Qian \\ Chongqing Key Laboratory of Emergency \\ Communication, Chongqing Communication Institute \\ Chongqing 400035, China \\ e-mail: charlige@126.com
}

words, Construction 2 can produce $N_{k}$ distinct PQAs. Due to the fact that the users' number in a system is decided by the number of perfect arrays employed, Construction 2 in [14] can provide lots of candidates for applications.

Incidentally, it should be noted that PQAs theory is fundamental, since constructions of perfect arrays over some constellations, such as 16-QAM constellation, depend on their results [16].

The remainder of this paper is organized as follows. In Sect. II, the necessary concepts are recalled. In Sect. III, Zeng, et al's constructions are briefly given. In the following section, the main results are stated. Two examples will appear in Sect. V. Finally, we conclude this paper in Sect. VI.

\section{BASIC CONCEPTS}

A $n$-dimensional function is said to be an array, denoted traditionally by

$$
A=\left[a_{i_{1}, i_{2}, \cdots, i_{n}} \mid 0 \leq i_{k} \leq N_{k}-1,1 \leq k \leq n\right],
$$

where $N_{1} \times \cdots \times N_{k-1} \times N_{k} \times N_{k+1} \times \cdots \times N_{n}$ is referred to as size.

For two arrays $A$ and $B$, we define their correlation function by

$$
\begin{aligned}
& R_{A, B}\left(\tau_{1}, \tau_{2}, \cdots, \tau_{n}\right) \\
& =\sum_{i_{1}=0}^{N_{1}-1} \sum_{i_{2}=0}^{N_{2}-1} \cdots \sum_{i_{n}=0}^{N_{n}-1} a_{i_{1}, i_{2}, \cdots, i_{n}} b_{i_{1}+\tau_{1}, i_{2}+\tau_{2}, \cdots, i_{n}+\tau_{n}}^{*},
\end{aligned}
$$

where the symbol "**" denotes the complex conjugate, and the addition " $i_{k}+\tau_{k}(1 \leq k \leq n)$ " is performed modulo $N_{k}$. If $A=B$, we call $R_{A, A}\left(\tau_{1}, \tau_{2}, \cdots, \tau_{n}\right)$ an periodic autocorrelation function, otherwise, a periodic crosscorrelation function.

If the autocorrelation function of an array $A$ satisfies 


$$
\begin{aligned}
& R_{A, A}\left(\tau_{1}, \tau_{2}, \cdots, \tau_{n}\right) \\
& = \begin{cases}\sum_{i_{1}=0}^{N_{1}-1} \sum_{i_{2}=0}^{N_{2}-1} \cdots \sum_{i_{n}=0}^{N_{n}-1}\left|a_{i_{1}, i_{2}, \cdots, i_{n}}\right|^{2} & \left(\tau_{1}, \cdots, \tau_{n}\right)=(0, \cdots, 0) \\
0 & \left(\tau_{1}, \cdots, \tau_{n}\right) \neq(0, \cdots, 0),\end{cases}
\end{aligned}
$$

we are referred to the array $A$ as a perfect array.

Let $T$ denote a cyclical shift operator, in a mathematical term, for a $n$-dimensional $A$ and $n$ integers $\mu_{k}$ 's $(1 \leq k \leq n)$, we have

$$
\begin{aligned}
& T\left(\mu_{1}, \mu_{2}, \cdots, \mu_{n}\right) A \\
& =\left[a_{i_{1}+\mu_{1}, i_{2}+\mu_{2}, \cdots, i_{n}+\mu_{n}} \mid 0 \leq i_{k} \leq N_{k}-1,1 \leq k \leq n\right],
\end{aligned}
$$

where the addition " $i_{k}+\mu_{k}(1 \leq k \leq n)$ " is performed modulo $N_{k}$.

For two arrays $A$ and $B$, if there are $n$ integers $\mu_{k}$ 's $(1 \leq k \leq n)$ so as to satisfy

$$
T\left(\mu_{1}, \mu_{2}, \cdots, \mu_{n}\right) A=B,
$$

we say that these two arrays are cyclical shift equivalence, otherwise, distinct arrays.

Let $A=\left[a_{i_{1}, i_{2}, \cdots, i_{n}}\right]$ and $B=\left[b_{i_{1}, i_{2}, \ldots, i_{n}}\right]$ be two binary arrays with size $N_{1} \times \cdots \times N_{k-1} \times N_{k} \times N_{k+1} \times \cdots$ $\times N_{n}$. We construct two quaternary arrays by

$Q^{l}=\left[q_{i_{1}, i_{2}, \cdots, i_{n}}^{l}\right](l=1,2)$

$q_{i_{1}, i_{2}, \cdots, i_{n}}^{l}=\phi_{l}\left(a_{i_{1}+\eta_{l 1}, i_{2}+\eta_{l 2}, \cdots, i_{n}+\eta_{l n}}, b_{i_{1}+\delta_{l l}, i_{2}+\delta_{l 2}, \cdots, i_{n}+\delta_{l n}}\right)$

$\phi_{1}(x, y)=\frac{1+j}{2}(-1)^{x}+\frac{1-j}{2}(-1)^{y}$

$\phi_{2}(x, y)=\frac{1+j}{2}(-1)^{x}-\frac{1-j}{2}(-1)^{y}$,

where $x, y \in\{0,1\}$, and $\eta_{l k}$ and $\delta_{l k}(1 \leq k \leq n)$ are integers.

\section{ZENG, ET AL'S CONSTRUCTIONS}

Zeng, et al gave two constructions in [14] so as to convert a PBA into a PQA. For the sake of saving the reader's trouble in referring to the relevant reference, we briefly state them below.

Construction 1 [14].

Consider a PBA $A$, namely, $A=B$, with size $N_{1} \times \cdots \times N_{k-1} \times N_{k} \times N_{k+1} \times \cdots \times N_{n} \quad$, where all integers $N_{k}$ 's $(1 \leq k \leq n)$ are even. If we have

$$
\eta_{l k} \equiv \delta_{l k}\left(\bmod \frac{N_{k}}{2}\right) \quad(1 \leq k \leq n),
$$

the resultant quaternary arrays $Q^{l}(l=1,2)$ in (6) is perfect.

Construction 2 [14].

Consider two PBAs $A$ and $B$, with size $N_{1} \times \cdots \times N_{k-1} \times N_{k} \times N_{k+1} \times \cdots \times N_{n}$, where at least an integer in the integers $N_{k}$ 's $(1 \leq k \leq n)$, say $N_{r}$, is odd.
We construct a quaternary array $P=\left[p_{i_{1}, \cdots, i_{r-1}, i_{r}, i_{r+1}, \cdots, i_{n}}\right]$ by

$$
p_{i_{1}, \cdots, i_{r-1}, i_{r}^{\prime}, i_{r+1}, \cdots, i_{n}}= \begin{cases}h_{i_{1}, \cdots, i_{r-1}, i_{r}, i_{r+1}, \cdots, i_{n}}^{1} & i_{r}^{\prime}=2 i_{r} \\ h_{i_{1}, \cdots, i_{r-1}, i_{r}, i_{r+1}, \cdots, i_{n}}^{2} & i_{r}^{\prime}=2 i_{r}+1,\end{cases}
$$

(8)

$$
\begin{aligned}
& \text { where } \\
& h_{i_{1}, \cdots, i_{r-1}, i_{r}, i_{r+1}, \cdots, i_{n}}^{1}=\phi_{1}\left(a_{i_{1}+\eta_{1}, \cdots, i_{r-1}+\eta_{r-1}, i_{r}+\eta_{r}, i_{r+1}+\eta_{r+1}, \cdots, i_{n}+\eta_{n}},\right. \\
& \left.b_{i_{1}+\delta_{1}, \cdots, i_{r-1}+\delta_{r-1}, i_{r}+\delta_{r}, i_{r+1}+\delta_{r+1}, \cdots, i_{n}+\delta_{n}}\right), \\
& h_{i_{1}, \cdots, i_{r-1}, i_{r}, i_{r+1}, \cdots, i_{n}}^{2}=\phi_{2}\left(a_{i_{1}+\xi_{1}, \cdots, i_{r-1}+\varsigma_{r-1}, i_{r}+\varsigma_{r}, i_{r+1}+\varsigma_{r+1}, \cdots, i_{n}+\varsigma_{n}},\right. \\
& \left.b_{i_{1}+\lambda_{1}, \cdots, i_{r-1}+\lambda_{r-1}, i_{r}+\lambda_{r}, i_{r+1}+\lambda_{r+1}, \cdots, i_{n}+\lambda_{n}}\right),
\end{aligned}
$$

and $\quad 0 \leq i_{k} \leq N_{k}-1(1 \leq k \leq n) \quad$ and $\quad i_{r}^{\prime}=2 i_{r}+t$ ( $t \in\{0,1\}$ ), that is, $0 \leq i_{r}^{\prime} \leq 2 N_{r}-1$. If we have

$$
\begin{cases}\delta_{l}-\eta_{l} \equiv \lambda_{l}-\varsigma_{l} & \bmod N_{l}(1 \leq l \leq n) \\ \varsigma_{l}-\delta_{l} \equiv \eta_{l}-\lambda_{l} & \bmod N_{l}(1 \leq l \leq n, l \neq r)(11) \\ \varsigma_{r}-\delta_{r} \equiv \eta_{r}-\lambda_{r}+1 & \bmod N_{r},\end{cases}
$$

the quaternary array $P$ with size $N_{1} \times \cdots \times N_{r-1} \times 2 N_{r}$ $\times N_{r+1} \times \cdots \times N_{n}$ is perfect.

Apparently, Theorem 2 does not give the family size of Construction 2.

\section{FAMILY Size OF CONSTRUCTION 2}

First of all, we need to investigate the conditions under which Construction 2 results in distinct arrays.

Let arrays $A$ and $B$ be two PBAs with each of size $N_{1} \times \cdots \times N_{r-1} \times N_{r} \times N_{r+1} \times \cdots \times N_{n} \quad$, where the integers $N_{r}$ is odd. Again let the array $P$ be produced by Eqs. (8)-(10) from the arrays $A$ and $B$ with integers $\eta_{l}$ 's, $\delta_{l}$ 's, $\varsigma_{l}$ 's, and $\lambda_{l}$ 's $(1 \leq l \leq n)$, and so does the array $P^{\prime}$ but with integers $\eta_{l}^{\prime}$ 's, $\delta_{l}^{\prime}$ 's, $\varsigma_{l}^{\prime}$ 's, and $\lambda_{l}^{\prime}$ 's $(1 \leq l \leq n)$. If the following conditions:

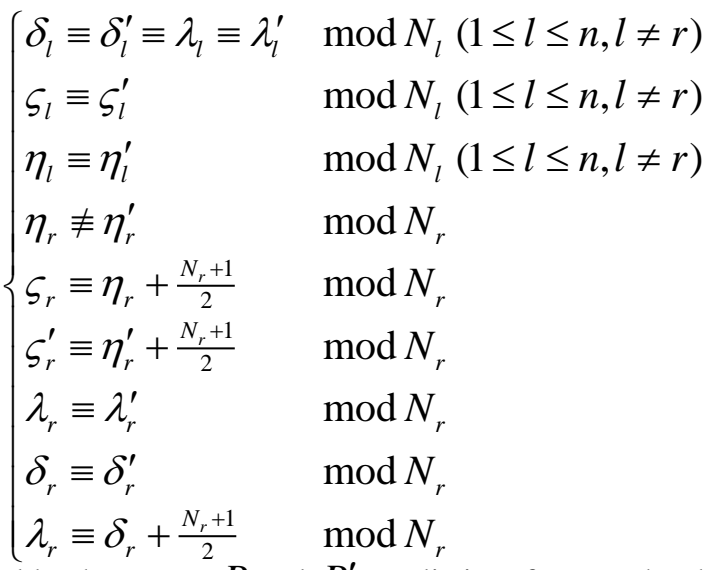

holds, the arrays $P$ and $P^{\prime}$ are distinct from each other.

Provided that there exist the integers $\mu_{l}$ 's $(1 \leq l \leq n)$ so that $T\left(\mu_{1}, \mu_{2}, \cdots, \mu_{n}\right) P=P^{\prime}$, which means that the arrays $P$ and $P^{\prime}$ are cyclical shift equivalence. In 
accordance with Eq. (8), the entries of the array $T\left(\mu_{1}, \mu_{2}, \cdots, \mu_{n}\right) P$ can be calculated by four cases as follows.

Case 1: $i_{r}^{\prime}=2 i_{r}$ and $\mu_{r}=2 \rho_{r}$.

$$
\begin{aligned}
p_{i_{1}+\mu_{1}, \cdots, i_{r-1}+\mu_{r-1}, i_{r}^{\prime}+\mu_{r}, i_{r+1}+\mu_{r+1}, \cdots, i_{n}+\mu_{n}}= \\
h_{i_{1}+\mu_{1}, \cdots, i_{r-1}+\mu_{r-1}, i_{r}+\rho_{r}, i_{r+1}+\mu_{r+1}, \cdots, i_{n}+\mu_{n}}^{1} .
\end{aligned}
$$

Case 2: $i_{r}^{\prime}=2 i_{r}+1$ and $\mu_{r}=2 \rho_{r}$.

$$
\begin{aligned}
& p_{i_{1}+\mu_{1}, \cdots, i_{r-1}+\mu_{r-1}, i_{r}^{\prime}+\mu_{r}, i_{r+1}+\mu_{r+1}, \cdots, i_{n}+\mu_{n}}= \\
& h_{i_{1}+\mu_{1}, \cdots, i_{r-1}+\mu_{r-1}, i_{r}+\rho_{r}, i_{r+1}+\mu_{r+1}, \cdots, i_{n}+\mu_{n}}^{2} .
\end{aligned}
$$

Case 3: $i_{r}^{\prime}=2 i_{r}$ and $\mu_{r}=2 \rho_{r}+1$.

$$
\begin{aligned}
p_{i_{1}+\mu_{1}, \cdots, i_{r-1}+\mu_{r-1}, i_{r}^{\prime}+\mu_{r}, i_{r+1}+\mu_{r+1}, \cdots, i_{n}+\mu_{n}} & = \\
& h_{i_{1}+\mu_{1}, \cdots, i_{r-1}+\mu_{r-1}, i_{r}+\rho_{r}, i_{r+1}+\mu_{r+1}, \cdots, i_{n}+\mu_{n}}^{2} .
\end{aligned}
$$

Case 4: $i_{r}^{\prime}=2 i_{r}+1$ and $\mu_{r}=2 \rho_{r}+1$.

$$
\begin{aligned}
& p_{i_{1}+\mu_{1}, \cdots, i_{r-1}+\mu_{r-1}, i_{r}^{\prime}+\mu_{r}, i_{r+1}+\mu_{r+1}, \cdots, i_{n}+\mu_{n}}= \\
& h_{i_{1}+\mu_{1}, \cdots, i_{r-1}+\mu_{r-1}, i_{r}+\rho_{r}+1, i_{r+1}+\mu_{r+1}, \cdots, i_{n}+\mu_{n}}^{1} .
\end{aligned}
$$

According to $T\left(\mu_{1}, \mu_{2}, \cdots, \mu_{n}\right) P=P^{\prime}$, for Cases 14 we have

$\phi_{1}\left(a_{i_{1}+\eta_{1}+\mu_{1}, \cdots, i_{r-1}+\eta_{r-1}+\mu_{r-1}, i_{r}+\eta_{r}+\rho_{r}, i_{r+1}+\eta_{r+1}+\mu_{r+1}, \cdots, i_{n}+\eta_{n}+\mu_{n}}\right.$,

$\left.b_{i_{1}+\delta_{1}+\mu_{1}, \cdots, i_{r-1}+\delta_{r-1}+\mu_{r-1}, i_{r}+\delta_{r}+\rho_{r}, i_{r+1}+\delta_{r+1}+\mu_{r+1}, \cdots, i_{n}+\delta_{n}+\mu_{n}}\right)=$

$\phi_{1}\left(a_{i_{1}+\eta_{1}^{\prime}, \cdots, i_{r}+\eta_{r}^{\prime}, \cdots, i_{n}+\eta_{n}^{\prime}}, b_{i_{1}+\delta_{1}^{\prime}, \cdots, i_{r}+\delta_{r}^{\prime}, \cdots, i_{n}+\delta_{n}^{\prime}}\right)$,

$\phi_{2}\left(a_{i_{1}+\varsigma_{1}+\mu_{1}, \cdots, i_{r-1}+\varsigma_{r-1}+\mu_{r-1}, i_{r}+\varsigma_{r}+\rho_{r}, i_{r+1}+\varsigma_{r+1}+\mu_{r+1}, \cdots, i_{n}+\varsigma_{n}+\mu_{n}}\right.$,

$\left.b_{i_{1}+\lambda_{1}+\mu_{1}, \cdots, i_{r-1}+\lambda_{r-1}+\mu_{r-1}, i_{r}+\lambda_{r}+\rho_{r}, i_{r+1}+\lambda_{r+1}+\mu_{r+1}, \cdots, i_{n}+\lambda_{n}+\mu_{n}}\right)=$ (18)

$\phi_{2}\left(a_{i_{1}+\varsigma_{1}^{\prime}, \cdots, i_{r}+\varsigma_{r}^{\prime}, \cdots, i_{n}+\varsigma_{n}^{\prime}}, b_{i_{1}+\lambda_{1}^{\prime}, \cdots, i_{r}+\lambda_{r}^{\prime}, \cdots, i_{n}+\lambda_{n}^{\prime}}\right)$,

$\phi_{2}\left(a_{i_{1}+\varsigma_{1}+\mu_{1}, \cdots, i_{r-1}+\varsigma_{r-1}+\mu_{r-1}, i_{r}+\varsigma_{r}+\rho_{r}, i_{r+1}+\varsigma_{r+1}+\mu_{r+1}, \cdots, i_{n}+\varsigma_{n}+\mu_{n}}\right.$,

$\left.b_{i_{1}+\lambda_{1}+\mu_{1}, \cdots, i_{r-1}+\lambda_{r-1}+\mu_{r-1}, i_{r}+\lambda_{r}+\rho_{r}, i_{r+1}+\lambda_{r+1}+\mu_{r+1}, \cdots, i_{n}+\lambda_{n}+\mu_{n}}\right)=$ (19)

$\phi_{1}\left(a_{i_{1}+\eta_{1}^{\prime}, \cdots, i_{r}+\eta_{r}^{\prime}, \cdots, i_{n}+\eta_{n}^{\prime}}, b_{i_{1}+\delta_{1}^{\prime}, \cdots, i_{r}+\delta_{r}^{\prime}, \cdots, i_{n}+\delta_{n}^{\prime}}\right)$,

and

$\phi_{1}\left(a_{i_{1}+\eta_{1}+\mu_{1}, \cdots, i_{r-1}+\eta_{r-1}+\mu_{r-1}, i_{r}+\eta_{r}+\rho_{r}+1, i_{r+1}+\eta_{r+1}+\mu_{r+1}, \cdots, i_{n}+\eta_{n}+\mu_{n}}\right.$,

$\left.b_{i_{1}+\delta_{1}+\mu_{1}, \cdots, i_{r-1}+\delta_{r-1}+\mu_{r-1}, i_{r}+\delta_{r}+\rho_{r}+1, i_{r+1}+\delta_{r+1}+\mu_{r+1}, \cdots, i_{n}+\delta_{n}+\mu_{n}}\right)=$

$\phi_{2}\left(a_{i_{1}+\varsigma_{1}^{\prime}, \cdots, i_{r}+\varsigma_{r}^{\prime}, \cdots, i_{n}+\varsigma_{n}^{\prime}}, b_{i_{1}+\lambda_{1}^{\prime}, \cdots, i_{r}+\lambda_{r}^{\prime}, \cdots, i_{n}+\lambda_{n}^{\prime}}\right)$,

respectively.

Consider $\mu_{r}$ even, which results in that Cases 1 and 2 appear in $T\left(\mu_{1}, \mu_{2}, \cdots, \mu_{n}\right) P=P^{\prime}$ synchronously. For Case 1, from Eqs. (6) and (17) we have

$$
\left[(-1)^{\alpha}-(-1)^{\gamma}\right]-j\left[(-1)^{\beta}-(-1)^{\theta}\right]=0,
$$

where

$$
\left\{\begin{array}{l}
\alpha=a_{i_{1}+\eta_{1}+\mu_{1}, \cdots, i_{r-1}+\eta_{r-1}+\mu_{r-1}, i_{r}+\eta_{r}+\rho_{r}, i_{r+1}+\eta_{r+1}+\mu_{r+1}, \cdots, i_{n}+\eta_{n}+\mu_{n}} \\
\beta=b_{i_{1}+\delta_{1}+\mu_{1}, \cdots, i_{r-1}+\delta_{r-1}+\mu_{r-1}, i_{r}+\delta_{r}+\rho_{r}, i_{r+1}+\delta_{r+1}+\mu_{r+1}, \cdots, i_{n}+\delta_{n}+\mu_{n}} \\
\gamma=a_{i_{1}+\eta_{1}^{\prime}, \cdots, i_{r}+\eta_{r}^{\prime}, \cdots, i_{n}+\eta_{n}^{\prime}} \\
\theta=b_{i_{1}+\delta_{1}^{\prime}, \cdots, i_{r}+\delta_{r}^{\prime}, \cdots, i_{n}+\delta_{n}^{\prime}} .
\end{array}\right.
$$

(22)

Hence, from Eq.(21) we have

Since the equation system in (23) holds for arbitrary integers $i_{l}$ 's $(1 \leq l \leq n)$, we must have

$$
\begin{cases}\eta_{l}+\mu_{l} \equiv \eta_{l}^{\prime} & \bmod N_{l}(1 \leq l \leq n, l \neq r) \\ \eta_{r}+\rho_{r} \equiv \eta_{r}^{\prime} & \bmod N_{r} \\ \delta_{l}+\mu_{l} \equiv \delta_{l}^{\prime} & \bmod N_{l}(1 \leq l \leq n, l \neq r) \\ \delta_{r}+\rho_{r} \equiv \delta_{r}^{\prime} & \bmod N_{r},\end{cases}
$$

which results in $\rho_{r} \equiv 0\left(\bmod N_{r}\right)$ and $\eta_{r} \equiv \eta_{r}^{\prime}$ $\left(\bmod N_{r}\right)$ under given conditions (e.g., $\delta_{r} \equiv \delta_{r}^{\prime}$ $\left.\left(\bmod N_{r}\right)\right)$ in Theorem 3. But, we have set $\eta_{r} \not \equiv \eta_{r}^{\prime}$ $\left(\bmod N_{r}\right)$ in the conditions of Theorem 3. Apparently, here is a contradiction.

Similarly, consider $\mu_{r}$ odd, which results in that Cases 3 and 4 appear in Eq. $T\left(\mu_{1}, \mu_{2}, \cdots, \mu_{n}\right) P=P^{\prime}$ synchronously. For Case 3, from Eqs. (6) and (19) we have

(25)

where

$\left\{\begin{array}{l}\alpha_{1}=a_{i_{1}+\varsigma_{1}+\mu_{1}, \cdots, i_{r-1}+\varsigma_{r-1}+\mu_{r-1}, i_{r}+\varsigma_{r}+\rho_{r}, i_{r+1}+\varsigma_{r+1}+\mu_{r+1}, \cdots, i_{n}+\varsigma_{n}+\mu_{n}} \\ \beta_{1}=b_{i_{1}+\lambda_{1}+\mu_{1}, \cdots, i_{r-1}+\lambda_{r-1}+\mu_{r-1}, i_{r}+\lambda_{r}+\rho_{r}, i_{r+1}+\lambda_{r+1}+\mu_{r+1}, \cdots, i_{n}+\lambda_{n}+\mu_{n}} \\ \gamma_{1}=a_{i_{1}+\eta_{1}^{\prime}, \cdots, i_{r}+\eta_{r}^{\prime}, \cdots, i_{n}+\eta_{n}^{\prime}} \\ \theta_{1}=b_{i_{1}+\delta_{1}^{\prime}, \cdots, i_{r}+\delta_{r}^{\prime}, \cdots, i_{n}+\delta_{n}^{\prime}} .\end{array}\right.$

(26)

Hence, from Eq. (25) we have

$$
(-1)^{\beta_{1}}=-(-1)^{\theta_{1}}
$$

In accordance with Eq. (12), from Eq. (27) we have

$$
\left\{\begin{array}{l}
\beta_{1}=b_{i_{1}+\delta_{1}+\mu_{1}, \cdots, i_{r-1}+\delta_{r-1}+\mu_{r-1}, i_{r}+\delta_{r}+\rho_{r}+\frac{N_{r}+1}{2}, i_{r+1}+\delta_{r+1}+\mu_{r+1}, \cdots, i_{n}+\delta_{n}+\mu_{n}} \\
\theta_{1}=b_{i_{1}+\delta_{1}, \cdots, i_{r}+\delta_{r}, \cdots, i_{n}+\delta_{n}} .
\end{array}\right.
$$

Notice the fact that for given integers $\delta_{l}$ 's $(1 \leq l \leq n)$, the array $B^{\prime}=\left[b_{i_{1}+\delta_{1}, \cdots, i_{r}+\delta_{r}, \cdots, i_{n}+\delta_{n}}\right]$ is perfect due to the perfect array $B$. On the other hand, we count the autocorrelation of the array $B^{\prime}$ as follows. 


$$
\begin{aligned}
& R_{B^{\prime}, B^{\prime}}\left(\mu_{1}, \cdots, \mu_{r-1}, \rho_{r}+\frac{N_{r}+1}{2}, \mu_{r+1}, \cdots, \mu_{n}\right) \\
& =\sum_{i_{1}=0}^{N_{1}-1} \sum_{i_{2}=0}^{N_{2}-1} \cdots \sum_{i_{n}=0}^{N_{n}-1}(-1)^{\theta_{1}}(-1)^{\beta_{1}} \\
& =-\sum_{i_{1}=0}^{N_{1}-1} \sum_{i_{2}=0}^{N_{2}-1} \cdots \sum_{i_{n}=0}^{N_{n}-1}\left[(-1)^{\theta_{1}}\right]^{2} \\
& =-N_{1} \times \cdots \times N_{n} \neq 0,
\end{aligned}
$$

where we employ Eq. (27). As a consequence, a contradiction gives rise to here. Apparently, the above derivation suggests that in $\mu_{r}$ odd case, the condition $\eta_{r} \not \equiv \eta_{r}^{\prime}\left(\bmod N_{r}\right)$ is not necessary.

Summarizing the above, we come to the conclusion that Theorem 3 is true.

The next theorem will answer the family size of Construction 2.

For a given PBA, Construction 2 yields $N_{r}$ distinct PQAs.

Proof: From Theorem 3, when $\eta_{r}$ ranges the range from 0 to $N_{r}-1$ with other parameters unaltered in Eq. (12), the obtained arrays resulting from Construction 2 are distinct from one another. We complete the proof of this theorem.

\section{EXAMPLES}

In order to help the reader understand our results, here are two examples.

\section{Example 1:}

Consider the arrays $A$ and $B$ be an identical PBA with size $3 \times 12$ [5] as follows.

$$
A=\left[\begin{array}{llllllllllll}
- & + & + & - & + & + & + & + & + & - & + & - \\
+ & + & + & + & - & + & + & - & + & - & + & - \\
+ & + & - & - & + & + & - & - & - & - & - & +
\end{array}\right] .
$$

Consider the odd integer $N_{1}=3$. According to Eq. (12) we set

$$
\left\{\begin{array}{l}
\left(\eta_{1}, \eta_{2}\right)=(\Gamma, 0) \\
\left(\delta_{1}, \delta_{2}\right)=(0,0) \\
\left(\varsigma_{1}, \varsigma_{2}\right)=(\Gamma+2,0) \\
\left(\lambda_{1}, \lambda_{2}\right)=(0,0),
\end{array}\right.
$$

Where $\Gamma=0,1,2$.

From Construction 2 the resultant distinct PQAs $P_{\Gamma}$ 's ( $\Gamma=0,1,2$ ) with size $6 \times 12$, depending on the choice of $\Gamma$, are given as follows, respectively.

$$
P_{0}=\left[\begin{array}{llllllllllll}
3 & 0 & 0 & 3 & 1 & 0 & 0 & 1 & 0 & 2 & 0 & 2 \\
0 & 1 & 2 & 3 & 1 & 1 & 2 & 2 & 2 & 3 & 2 & 0 \\
0 & 0 & 1 & 1 & 3 & 0 & 1 & 2 & 1 & 2 & 1 & 3 \\
2 & 1 & 1 & 2 & 0 & 1 & 1 & 0 & 1 & 3 & 1 & 3 \\
1 & 0 & 3 & 2 & 0 & 0 & 3 & 3 & 3 & 2 & 3 & 1 \\
1 & 1 & 0 & 0 & 2 & 1 & 0 & 3 & 0 & 3 & 0 & 2
\end{array}\right],
$$

$$
P_{1}=\left[\begin{array}{llllllllllll}
0 & 0 & 0 & 0 & 2 & 0 & 0 & 2 & 0 & 2 & 0 & 2 \\
3 & 1 & 1 & 3 & 1 & 1 & 1 & 1 & 1 & 3 & 1 & 3 \\
0 & 0 & 2 & 2 & 0 & 0 & 2 & 2 & 2 & 2 & 2 & 0 \\
1 & 1 & 1 & 1 & 3 & 1 & 1 & 3 & 1 & 3 & 1 & 3 \\
2 & 0 & 0 & 2 & 0 & 0 & 0 & 0 & 0 & 2 & 0 & 2 \\
1 & 1 & 3 & 3 & 1 & 1 & 3 & 3 & 3 & 3 & 3 & 1
\end{array}\right],
$$

and

$$
P_{2}=\left[\begin{array}{llllllllllll}
0 & 0 & 3 & 3 & 1 & 0 & 3 & 2 & 3 & 2 & 3 & 1 \\
0 & 1 & 1 & 0 & 2 & 1 & 1 & 2 & 1 & 3 & 1 & 3 \\
3 & 0 & 1 & 2 & 0 & 0 & 1 & 1 & 1 & 2 & 1 & 3 \\
1 & 1 & 2 & 2 & 0 & 1 & 2 & 3 & 2 & 3 & 2 & 0 \\
1 & 0 & 0 & 1 & 3 & 0 & 0 & 3 & 0 & 2 & 0 & 2 \\
2 & 1 & 0 & 3 & 1 & 1 & 0 & 0 & 0 & 3 & 0 & 2
\end{array}\right],
$$

the periodic autocorrelation function of whose each is depicted by Figure 1. Apparently, this function is impulselike.

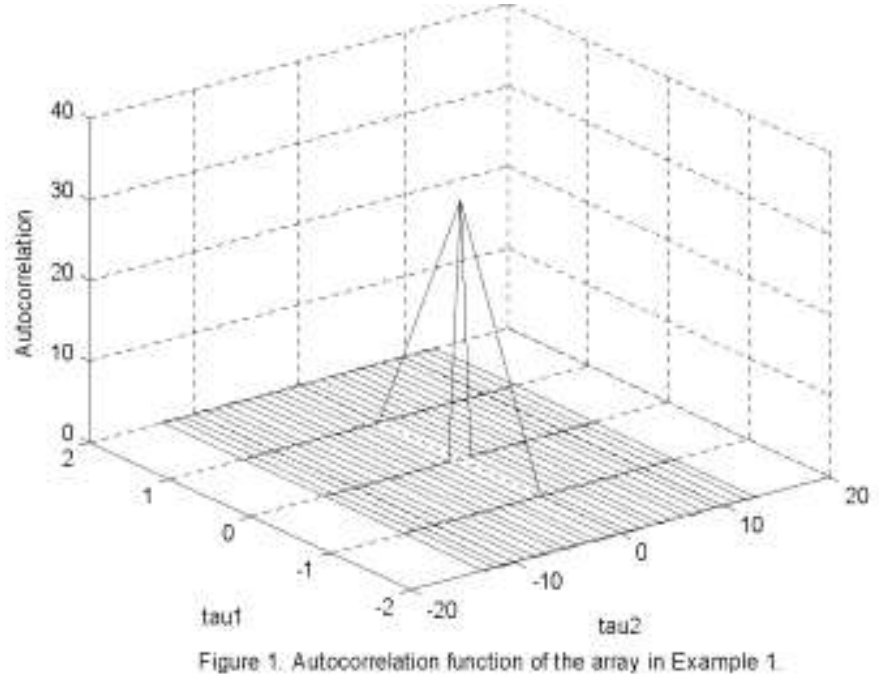

Example 2:

Consider the arrays $A$ and $B$ be an identical PBA with size $4 \times 3 \times 3$ [5] as follows. 


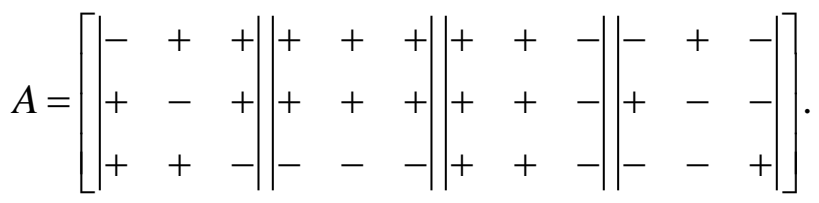

Consider the odd integer $N_{3}=3$. According to Eq. (12) we set

$$
\left\{\begin{array}{l}
\left(\eta_{1}, \eta_{2}, \eta_{3}\right)=(0,0, \Gamma) \\
\left(\delta_{1}, \delta_{2}, \delta_{3}\right)=(0,0,1) \\
\left(\varsigma_{1}, \varsigma_{2}, \varsigma_{3}\right)=(0,0, \Gamma+2) \\
\left(\lambda_{1}, \lambda_{2}, \lambda_{3}\right)=(0,0,0),
\end{array}\right.
$$

Where $\Gamma=0,1,2$.

In accordance with Construction 2, the resultant distinct PQAs $P_{\Gamma}$ 's $(\Gamma=0,1,2)$ with size $4 \times 3 \times 6$, depending on the choice of $\Gamma$, are given as follows, respectively.

$$
\begin{aligned}
& P_{0}=\left[\left|\begin{array}{llllll}
3 & 0 & 0 & 2 & 1 & 1 \\
1 & 1 & 3 & 0 & 0 & 2 \\
0 & 2 & 1 & 1 & 3 & 0
\end{array}\right|\left|\begin{array}{llllll}
0 & 1 & 0 & 1 & 0 & 1 \\
0 & 1 & 0 & 1 & 0 & 1 \\
2 & 3 & 2 & 3 & 2 & 3
\end{array}\right|\right. \\
& \left.\left|\begin{array}{llllll}
0 & 2 & 1 & 1 & 3 & 0 \\
0 & 2 & 1 & 1 & 3 & 0 \\
0 & 2 & 1 & 1 & 3 & 0
\end{array}\right|\left|\begin{array}{llllll}
3 & 3 & 1 & 2 & 2 & 0 \\
1 & 2 & 2 & 0 & 3 & 3 \\
2 & 0 & 3 & 3 & 1 & 2
\end{array}\right|\right] \\
& P_{1}=\left[\begin{array}{llllll}
0 & 3 & 0 & 1 & 2 & 1 \\
2 & 1 & 0 & 3 & 0 & 1 \\
0 & 1 & 2 & 1 & 0 & 3
\end{array}|| \begin{array}{llllll}
0 & 1 & 0 & 1 & 0 & 1 \\
0 & 1 & 0 & 1 & 0 & 1 \\
2 & 3 & 2 & 3 & 2 & 3
\end{array} \mid\right. \\
& \left.\left|\begin{array}{llllll}
0 & 1 & 2 & 1 & 0 & 3 \\
0 & 1 & 2 & 1 & 0 & 3 \\
0 & 1 & 2 & 1 & 0 & 3
\end{array}\right|\left|\begin{array}{llllll}
0 & 3 & 2 & 1 & 2 & 3 \\
2 & 1 & 2 & 3 & 0 & 3 \\
2 & 3 & 0 & 3 & 2 & 1
\end{array}\right|\right],
\end{aligned}
$$

and

$$
\begin{aligned}
& P_{2}=\left[\begin{array}{llllll}
0 & 0 & 3 & 1 & 1 & 2 \\
1 & 2 & 0 & 0 & 3 & 1 \\
3 & 1 & 1 & 2 & 0 & 0
\end{array}|| \begin{array}{llllll}
0 & 1 & 0 & 1 & 0 & 1 \\
0 & 1 & 0 & 1 & 0 & 1 \\
2 & 3 & 2 & 3 & 2 & 3
\end{array} \mid\right. \\
&\left.\left|\begin{array}{llllll}
3 & 1 & 1 & 2 & 0 & 0 \\
3 & 1 & 1 & 2 & 0 & 0 \\
3 & 1 & 1 & 2 & 0 & 0
\end{array}\right|\left|\begin{array}{llllll}
3 & 0 & 2 & 2 & 1 & 3 \\
2 & 2 & 1 & 3 & 3 & 0 \\
1 & 3 & 3 & 0 & 2 & 2
\end{array}\right|\right],
\end{aligned}
$$

the periodic autocorrelation function of whose each is

$$
\begin{aligned}
& R\left(\tau_{1}, \tau_{2}, \tau_{3}\right)= \\
& {\left[\begin{array}{llllll}
72 & 0 & 0 & 0 & 0 & 0 \\
0 & 0 & 0 & 0 & 0 & 0 \\
0 & 0 & 0 & 0 & 0 & 0
\end{array}|| \begin{array}{llllll}
0 & 0 & 0 & 0 & 0 & 0 \\
0 & 0 & 0 & 0 & 0 & 0 \\
0 & 0 & 0 & 0 & 0 & 0
\end{array} \mid\right.} \\
& \left.\left|\begin{array}{llllll}
0 & 0 & 0 & 0 & 0 & 0 \\
0 & 0 & 0 & 0 & 0 & 0 \\
0 & 0 & 0 & 0 & 0 & 0
\end{array}\right|\left|\begin{array}{llllll}
0 & 0 & 0 & 0 & 0 & 0 \\
0 & 0 & 0 & 0 & 0 & 0 \\
0 & 0 & 0 & 0 & 0 & 0
\end{array}\right|\right],
\end{aligned}
$$

where $0 \leq \tau_{1} \leq 3,0 \leq \tau_{2} \leq 2$, and $0 \leq \tau_{3} \leq 5$.

Apparently, as predicted, Eq. (36) shows that $P_{0}, P_{1}$, and $P_{2}$ are perfect. In addition, it is not difficult for the reader to check up that the arrays are distinct from one another.

\section{CONCLUSION}

This paper discusses the enumeration of Construction 2 in Zeng, et al's constructions, and gives the conditions that the resultant arrays are distinct. By the obtained conditions, more PQAs than the original theorem are produced. These proposed PQAs provide lots of candidates for applications

\section{ACKNOWLEDGMENT}

This work was supported by the National Natural Science Foundation of China (NSFC) under Grants 60872164, 61002034, 61171089, and 61271003.

\section{REFERENCES}

[1] P. Z. Fan and M. Darnell, Sequence design for communications applications. New York: John Wiley \& Sons Inc., 1996, pp.297 310.

[2] H. D. Lüke, "Sets of one and higher dimensional Welti codes and omplementary codes," IEEE Trans. Aerosp. and Electron. Syst. vol.AES-21, no.2, pp.170-179, May 1985.

[3] H. D. Lüke, "Sequences and arrays with perfect periodic correlation," IEEE Trans. Aerosp. and Electron. Syst., vol.24, no.3, pp.287-294, May 1985.

[4] H. D. Lüke, L. Bömer and M. Antweile, "Perfect binary arrays," Signal Process., vol.17, no.1, pp.69-80, May 1989.

[5] L. Bömer and M. Antweiler, "Perfect binary arrays with 36 elements," Electron. Lett., vol.23, no.14, pp.730-732, Jul. 1987.

[6] L. Bömer and M. Antweiler, "Two-dimensional perfect binary arrays with 64 elements," IEEE Trans. Inf. Theory, vol.36, no.2, pp.411-414, Mar. 1990

[7] D. Calabro and J. K. Wolf, "On the synthesis of two-dimensional arrays wirh diesirable properties," Inf. \& Contr., vol.11, pp.537560, 1976.

[8] L. E. Kopilovich, "On perfect binary arrays," Electron. Lett., vol.24, no.9, pp.566-567, Apr. 1988.

[9] J. Jedwab, C. Mitchell, F. Piper and P. Wild, "Perfect binary arrays and difference sets," Discr. Math., vol.125, No.1-3, pp. 241-254, Feb. 1994.

[10] L. Bömer and M. Antweiler, "Perfect three-level and three-phase sequences and arrays," IEEE Trans. Commun., vol.42, no.2/3/4, pp.767-772, Feb./Mar./Apr. 1994.

[11] M. Antweiler, L. Bömer and H. D. Lüke , "Perfect ternary arrays,' IEEE Trans. Inf. Theory, vol.36, no.3, pp.696-705, May 1990. 
[12] L. Bömer and M. Antweiler, "Perfect N-phase sequences and arrays," IEEE J. Selected areas in Commun., vol.310, no.4, pp.782-789, Mar. 1992.

[13] K. T. Arasu and Warwick de Launey, "Two-dimensional perfect quaternary arrays," IEEE Trans. on Inf. Theory, vol.47, no.4, pp 1482-1493, May 2001.

[14] F. X. Zeng, X. P. Zeng, Z. Y. Zhang, and G. X. Xuan, "Perfect quaternary arrays based on perfect binary arrays," WAP Conference Series: Information Science and Technology, vol.1 (April,2012), Proc. of 2011 International Conference on Information Science and Engineering (ICISE2011), Yangzhou, China, Sept. 29-Oct. 1, 2011, pp.444-448.
[15] K. T. Arasu, "Sequences and Arrays with Desirable Correlation Properties," In: NATO volume on Information Security, Coding Theory and Related Combinatorics., Ed. D. Crnkovic and V. Tonchev, pp.136-171, 2011.

[16] F. X. Zeng, X. P. Zeng, Z. Y. Zhang, and G. X. Xuan, "Perfect 16QAM sequences and arrays," IEICE Trans. Fundamentals, vol.E95-A, no.10, pp.1740-1748, Oct. 2012 